\title{
Viscosity solutions to second order parabolic PDEs on Riemannian manifolds
}

\author{
Xuehong Zhu
}

June 7, 2010

\begin{abstract}
In this work we consider viscosity solutions to second order parabolic PDEs $u_{t}+F\left(t, x, u, d u, d^{2} u\right)=0$ defined on compact Riemannian manifolds with boundary conditions. We prove comparison, uniqueness and existence results for the solutions. Under the assumption that the manifold $M$ has nonnegative sectional curvature, we get the finest results. If one additionally requires $F$ to depend on $d^{2} u$ in a uniformly continuous manner, the assumptions on curvature can be thrown away.
\end{abstract}

Keywords: Second order parabolic PDEs;Riemannian manifold; Viscosity solution.

\section{Introduction}

Since the theory of viscosity solutions was introduced by M. G. Crandall and P. L. Lions in the 1980's, it has been found that it's a very natural concept for a generalization of classical solutions. This theory has been applied widely and was enriched and expanded by many mathematicians. We would refer the reader to [1] and the references therein.

It should be natural to generalize the theory to problems on Riemannian manifolds since many functions arise from geometrical problems. But little is known about this field. Until recently, D. Azagra, J. Ferrera and B. Sanz[2] gave a work about Dirichlet problem on a complete Riemannian manifold with some restrictions on curvature. Almost at the same time, a few results about parabolic PDEs on Riemannian manifolds without boundary conditions were given in the appendix of [3].

We consider the following Cauchy-Dirichlet problem of the form: 


$$
\begin{cases}(E) & u_{t}+F\left(t, x, u, d u, d^{2} u\right)=0 \text { in }(0, T) \times \Omega \\ (B C) & u(t, x)=h(t, x),(t, x) \in[0, T) \times \partial \Omega \\ (I C) & u(0, x)=\psi(x), x \in \bar{\Omega}\end{cases}
$$

where $u$ is a function of $(t, x):[0, T) \times M \rightarrow R$ and $M$ is finite-dimensional complete Riemannian manifold. $d u, d^{2} u$ mean $d_{x} u(t, x)$ and $d_{x}^{2} u(t, x)$. $\Omega$ in $M$ is open and bounded. $T>0, h \in C([0, T) \times \bar{\Omega})$ and $\psi \in C(\bar{\Omega})$ are given.

Since there are not many intrinsic differences between elliptic and parabolic PDEs on manifolds, through an important theorem which is a Riemannian version of a result in Euclidean space in [1], we can get several comparison and uniqueness results which are parabolic versions of that in [2].

When we are faced with the existence of viscosity solutions, Perron's method is a good choice. Detailed research has been done in [1] about Dirichlet problem in Euclidean space. We will generalize two lemmas in [1] to parabolic PDEs on Riemannian manifolds and then get our existence result.

The next section is about the properties of second order parabolic viscosity subdifferentials on Riemannian manifolds and an important theorem for the proofs of comparison results; Section 3 are devoted to comparison results of our PDEs (1.1); Finally we prove the existence result of (1.1).

\section{Second order parabolic viscosity subdifferen- tials on Riemmanian manifolds}

Definition 2.1. Let $M$ be a finite-dimensional Riemannian manifold, and $f$ : $(0, T) \times M \rightarrow R$ a lower semicontinuous function. We define the second order parabolic subjet of $f$ at a point $(t, x) \in(0, T) \times M$ by

$$
\begin{aligned}
& \mathcal{P}^{2,-} f(t, x) \\
& =\left\{\left(\varphi_{t}(t, x), d \varphi(t, x), d^{2} \varphi(t, x)\right): \varphi \in C^{1,2}([0, T] \times M)\right. \text {, } \\
& f-\varphi \text { attains a local minimum } 0 \text { at }(t, x)\} \text {. }
\end{aligned}
$$

If $(p, \zeta, A) \in \mathcal{P}^{2,-} f(t, x)$, we will say that at the point $(t, x), p$ is a first order subdifferential of $f$ w.r.t. $t, \zeta$ is a first order subdifferential of $f$ w.r.t. $x$ and $A$ is a second order subdifferential of $f$ w.r.t. $x$.

Similarly, for an upper semicontinuous function $f:(0, T) \times M \rightarrow R$, we define the second order parabolic superjet of $f$ at a point $(t, x)$ by

$$
\begin{aligned}
& \mathcal{P}^{2,+} f(t, x) \\
& =\left\{\left(\varphi_{t}(t, x), d \varphi(t, x), d^{2} \varphi(t, x)\right): \varphi \in C^{1,2}([0, T] \times M)\right. \text {, } \\
& f-\varphi \text { attains a local maximum } 0 \text { at }(t, x)\} \text {. }
\end{aligned}
$$


Observe that $\mathcal{P}^{2,-} f(t, x)$ and $\mathcal{P}^{2,+} f(t, x)$ are subsets of $R \times T M_{x}^{*} \times \mathcal{L}_{s}^{2}\left(T M_{x}\right)$, where $T M_{x}^{*}$ stands for the cotangent space of $M$ at a point $x, T M_{x}$ stands for the tangent space at $x$ and $\mathcal{L}_{s}^{2}\left(T M_{x}\right)$ denotes the symmetric bilinear forms on $T M_{x}$. It is also clear that $\mathcal{P}^{2,-} f(t, x)=-\mathcal{P}^{2,+}(-f)(t, x)$.

In the sequel $M$ will always denotes an $n$-dimensional Riemannian manifold. We need the following several results for subjets which also hold, with obvious modification, for superjets.

Proposition 2.2. Let $f:(0, T) \times M \rightarrow R$ be a lower semicontinuous function. Let $p \in R, \zeta \in T M_{x}^{*}, A \in \mathcal{L}_{s}^{2}\left(T M_{x}\right),(t, x) \in(0, T) \times M$. The following statements are equivalent:

(i) $(p, \zeta, A) \in \mathcal{P}^{2,-} f(t, x)$.

(ii) $f\left(s, \exp _{x}(v)\right) \geq f(t, x)+p(s-t)+\langle\zeta, v\rangle_{x}+\frac{1}{2}\langle A v, v\rangle_{x}+o\left(|s-t|+\|v\|^{2}\right)$, as $|s-t| \rightarrow 0,\|v\| \rightarrow 0$.

Remark 2.3. This result is mainly due to [2] with a slight modification: $f$ is also a function of time $t$. Note that if $\varphi(t, x):(0, T) \times M \rightarrow R$ is differentialble w.r.t $t$ in $(0, T)$. Set $\psi(t, v)=\varphi\left(t, \exp _{x}(v)\right)$, where $v$ is valued on a neighborhood of $0_{x}$ in $T M_{x}$. Then $\varphi_{t}(s, y)=\psi_{t}\left(s, \exp _{x}^{-1}(y)\right)$. So obviously we can use almost the same method as Proposition 2.2 in [2] to get the proof of our proposition. The following Corollary 2.4 and Proposition 2.6 are also analogues of that in [2]. So we omit the proofs.

Proposition 2.4. Let $f:(0, T) \times M \rightarrow R$ be a lower semicontinuous function, and consider $p \in R, \zeta \in T M_{x}^{*}, A \in \mathcal{L}_{s}^{2}\left(T M_{x}\right),(t, x) \in(0, T) \times M$. Set $\bar{f}(t, v)=$ $f\left(t, \exp _{x}(v)\right)$. Then

$$
(p, \zeta, A) \in \mathcal{P}^{2,-} f(t, x) \Leftrightarrow(p, \zeta, A) \in \mathcal{P}^{2,-} \bar{f}\left(t, 0_{x}\right) .
$$

Definition 2.5. Let $f:(0, T) \times M \rightarrow R$ be a lower semicontinuous function, and $(t, x) \in(0, T) \times M$. We define

$$
\begin{aligned}
\overline{\mathcal{P}}^{2,-} f(t, x)=\{ & (p, \zeta, A) \in R \times T M_{x}^{*} \times \mathcal{L}_{s}^{2}\left(T M_{x}\right): \\
& \exists\left(t_{n}, x_{n}, p_{n}, \zeta_{n}, A_{n}\right) \in(0, T) \times M \times R \times T M_{x_{n}}^{*} \times \mathcal{L}_{s}^{2}\left(T M_{x_{n}}\right), \\
& \text { s.t. }\left(p_{n}, \zeta_{n}, A_{n}\right) \in \mathcal{P}^{2,-} f\left(t_{n}, x_{n}\right), \\
& \left.\left(t_{n}, x_{n}, f\left(t_{n}, x_{n}\right), p_{n}, \zeta_{n}, A_{n}\right) \rightarrow(t, x, f(t, x), p, \zeta, A)\right\} .
\end{aligned}
$$

and for an upper semicontinuous function $f(t, x)$ defined on $(0, T) \times M$ we define $\overline{\mathcal{P}}^{2,+} f(t, x)$ in the obvious way.

Proposition 2.6. Let $f:(0, T) \times M \rightarrow R$ be a lower semicontinuous function, and consider $p \in R, \zeta \in T M_{x}^{*}, A \in \mathcal{L}_{s}^{2}\left(T M_{x}\right),(t, x) \in(0, T) \times M$. Set $\bar{f}(t, v)=$ $f\left(t, \exp _{x}(v)\right)$. Then

$$
(p, \zeta, A) \in \overline{\mathcal{P}}^{2,-} f(t, x) \Leftrightarrow(p, \zeta, A) \in \overline{\mathcal{P}}^{2,-} \bar{f}\left(t, 0_{x}\right) .
$$


The following result is the Riemannian version of Theorem 8.3 in [1] and, as in that paper, will be the key to the proofs of comparison and uniqueness results for viscosity solution of second order parabolic PDEs on Riemannian manifolds. For the proof, see [3, Theorem 3.8].

Theorem 2.7. Let $M_{1}, M_{2}, \ldots M_{k}$ be Riemannian manifolds, and $\Omega_{i} \subset M_{i}$ open subsets. Define $\Omega=\Omega_{1} \times \ldots \times \Omega_{k} \subset M_{1} \times \ldots \times M_{k}=M$. Let $u_{i}$ be upper semicontinuous functions on $(0, T) \times \Omega_{i}, i=1,2, \ldots k$; let $\varphi$ be in $C^{1,2}((0, T) \times \Omega)$ and set

$$
w(t, x)=u_{1}\left(t, x_{1}\right)+\cdots+u_{k}\left(t, x_{k}\right)-\varphi(t, x),
$$

for $t \in(0, T), x=\left(x_{1}, \ldots, x_{k}\right) \in \Omega$. Assume that $(\hat{t}, \hat{x})=\left(\hat{t}, \hat{x}_{1}, \ldots, \hat{x}_{k}\right) \in(0, T) \times \Omega$ s.t.

$$
w\left(t, x_{1}, \ldots, x_{k}\right) \leq w\left(\hat{t}, \hat{x}_{1}, \ldots, \hat{x}_{k}\right), \text { for } t \in(0, T), x_{i} \in \Omega_{i} .
$$

Assume, moreover, that there is an $\delta>0$ s.t. for every $N>0$ there is a $C$ s.t. for $i=1, \ldots, k$

$$
\begin{aligned}
& p_{i} \leq C \text { whenever }\left(p_{i}, \zeta_{i}, A_{i}\right) \in \mathcal{P}^{2,+} u_{i}\left(t, x_{i}\right) \\
& d\left(x_{i}, \hat{x}_{i}\right)+|t-\hat{t}| \leq \delta \text { and }\left|u_{i}\left(t, x_{i}\right)\right|+\left\|\zeta_{i}\right\|+\left\|A_{i}\right\| \leq N
\end{aligned}
$$

where $d(\cdot, \cdot)$ denotes the Riemannian distance in $M$.

Then for each $\varepsilon>0$, there are $B_{i} \in \mathcal{L}_{s}^{2}\left(\left(T M_{i}\right)_{\hat{x}_{i}}\right)$ such that

$$
\left\{\begin{array}{c}
\left(p_{i}, d_{x_{i}} \varphi\left(\hat{t}, \hat{x}_{1}, \ldots, \hat{x}_{k}\right), B_{i}\right) \in \overline{\mathcal{P}}^{2,+} u_{i}\left(\hat{t}, \hat{x}_{i}\right) \text { for } i=1, \ldots, k \\
-\left(\frac{1}{\varepsilon}+\|A\|\right) I \leq\left(\begin{array}{ccc}
B_{1} & \cdots & 0 \\
\vdots & \ddots & \vdots \\
0 & \cdots & B_{k}
\end{array}\right) \leq A+\varepsilon A^{2} \\
p_{1}+\cdots+p_{k}=\varphi_{t}\left(\hat{t}, \hat{x}_{1}, \ldots, \hat{x}_{k}\right)
\end{array}\right.
$$

where $A=d^{2} \varphi(\hat{t}, \hat{x}) \in \mathcal{L}_{s}^{2}\left(T M_{\hat{x}}\right)$.

Now we extend the notion of viscosity solution to a parabolic equation on a Riemannian manifold. In the sequel we will denote

$$
\chi:=\left\{(t, x, r, \zeta, A): t \in[0, T], x \in M, r \in R, \zeta \in T M_{x}^{*}, A \in \mathcal{L}_{s}^{2}\left(T M_{x}\right)\right\} .
$$

Definition 2.8 (Viscosity solution). Let $M$ be a Riemannian manifold, and $F \in C(\chi, R)$. We say that an upper(lower) semicontinuous function $u$ : $[0, T) \times \bar{\Omega} \rightarrow R$ is a viscosity subsolution(supersolution) of $(1.1)$ on $[0, T) \times \bar{\Omega}$, if $u(t, x) \leq(\geq) h(t, x)$ for $0 \leq t<T$ and $x \in \partial \Omega$ and $u(0, x) \leq(\geq) \psi(x)$ for $x \in \bar{\Omega}$. Moreover, for all $(t, x) \in(0, T) \times \Omega$ and $(p, \zeta, A) \in \mathcal{P}^{2,+} u(t, x)\left(\mathcal{P}^{2,-} u(t, x)\right)$, $p+F(t, x, u(t, x), \zeta, A) \leq(\geq) 0$.

If $u$ is both a viscosity subsolution and a vicosity supersolution of (1.1), we say that $u$ is a viscosity solution of (1.1). 
Definition 2.9 (Degenerate ellipticity). Let $L_{x y}$ denote the parallel transport along the unique minimizing geodesic connecting $x$ to $y$ (assuming $x$ is close enough to $y$ so that this makes sense). This mapping is an isometry from $T M_{x}$ onto $T M_{y}$ (with inverse $L_{y x}$ ), and it induces an isometry (which we will still denote by $\left.L_{x y}\right), T M_{x}^{*} \ni \zeta \rightarrow L_{x y} \zeta \in T M_{y}^{*}$, defined by

$$
\left\langle L_{x y} \zeta, v\right\rangle_{y}:=\left\langle\zeta, L_{y x} v\right\rangle_{x} .
$$

Similarly, $L_{x y}$ induces an isometry $\mathcal{L}^{2}\left(T M_{x}\right) \ni A \rightarrow L_{x y}(A) \in \mathcal{L}^{2}\left(T M_{y}\right)$ defined by

$$
\left\langle L_{x y}(A) v, v\right\rangle_{y}:=\left\langle A\left(L_{y x} v\right), L_{y x} v\right\rangle_{x}
$$

where $\mathcal{L}^{2}\left(T M_{x}\right)$ denotes the space of bilinear forms on $T M_{x}$.

We will say that a function $F \in C(\chi, R)$ is degenerate elliptic provided that

$$
P \leq Q \Rightarrow F(x, r, \zeta, Q) \leq F(x, r, \zeta, P),
$$

for all $x \in M, r \in R, \zeta \in T M_{x}^{*}, P \in \mathcal{L}_{s}^{2}\left(T M_{x}\right), Q \in \mathcal{L}_{s}^{2}\left(T M_{x}\right)$.

Definition 2.10 (Properness). We will say that a function $F: M \times R \times$ $T M^{*} \times T_{2, s}(M) \rightarrow R,(x, r, \zeta, A) \rightarrow F(x, r, \zeta, A)$, is proper provided

(i) $F$ is degenerate elliptic, and

(ii) $F$ is nondecreasing in the variable $r$.

\section{Comparison results}

In this section and throughout the rest of the paper we will often abbreviate saying that $u$ is an upper semicontinuous function on a set $[0, T) \times \bar{\Omega}$ by writing $u \in U S C([0, T) \times \bar{\Omega})$. Similarly, $\operatorname{LSC}([0, T) \times \bar{\Omega})$ will stand for the set of lower semicontinuous functions on $[0, T) \times \bar{\Omega}$.

We denote by $i_{M}(x)$ injectivity radius of $M$ at $x$, that is the supremum of the radius $r$ of all balls $B\left(0_{x}, r\right)$ in $T M_{x}$ for which $\exp _{x}$ is a diffeomorphism from $B\left(0_{x}, r\right)$ onto $B(x, r)$. Similarly, $i(M)$ will denote the global injectivity radius of $M$, that is $i(M)=\inf \left\{i_{M}(x): x \in M\right\}$.

Theorem 3.1. Let $\Omega$ be a bounded open subset of a complete finite-dimensional Riemannian manifold $M$, and for each fixed $t \in(0, T), F \in C(\chi, R)$ be continuous, proper and satisfy: there exists a function $\omega:[0,+\infty] \rightarrow[0,+\infty]$ with $\omega(0+)=0$ and such that

$$
F\left(t, y, r, \alpha \exp _{y}^{-1}(x), Q\right)-F\left(t, x, r,-\alpha \exp _{x}^{-1}(y), P\right) \leq \omega\left(\alpha d^{2}(x, y)+d(x, y)\right),
$$


for all fixed $t \in(0, T)$ and for all $x, y \in \Omega, r \in R, P \in \mathcal{L}_{s}^{2}\left(T M_{x}\right), Q \in \mathcal{L}_{s}^{2}\left(T M_{y}\right)$ with

$$
-\left(\frac{1}{\varepsilon_{\alpha}}+\left\|A_{\alpha}\right\|\right)\left(\begin{array}{cc}
I & 0 \\
0 & I
\end{array}\right) \leq\left(\begin{array}{cc}
P & 0 \\
0 & -Q
\end{array}\right) \leq A_{\alpha}+\varepsilon_{\alpha} A_{\alpha}^{2},
$$

where $A_{\alpha}$ is the second derivative of the function $\varphi_{\alpha}(x, y)=\frac{\alpha}{2} d^{2}(x, y)$ at the point $(x, y) \in M \times M$,

$$
\varepsilon_{\alpha}=\frac{1}{2\left(1+\left\|A_{\alpha}\right\|\right)}
$$

and the points $x, y$ are assumed to be close enough to each other so that $d(x, y)<$ $\min \left\{i_{M}(x), i_{M}(y)\right\}$.

Let $u \in U S C([0, T) \times \bar{\Omega})$ be a subsolution and $v \in L S C([0, T) \times \bar{\Omega})$ a supersolution of (1.1). Then $u \leq v$ on $[0, T) \times \Omega$.

In particular the Cauchy-Dirichlet problem (1.1) has at most one viscosity solution.

Proof. We first observe that for $\varepsilon>0, \tilde{u}=u-\frac{\varepsilon}{T-t}$ is a vicosity subsolution of the problem with the form

$$
\left\{\begin{array}{l}
\tilde{u}_{t}+F\left(t, x, \tilde{u}+\frac{\varepsilon}{T-t}, d \tilde{u}, d^{2} \tilde{u}\right)=-\frac{\varepsilon}{(T-t)^{2}} \text { in }(0, T) \times \Omega, \\
\tilde{u}(t, x)=h(t, x)+\frac{\varepsilon}{t-T},(t, x) \in[0, T) \times \partial \Omega \\
u(0, x)=\psi(x)-\frac{\varepsilon}{T}, x \in \bar{\Omega} \\
\lim _{t \uparrow T} \tilde{u}(t, x)=-\infty \text { uniformly on } \bar{\Omega} .
\end{array}\right.
$$

Since $u \leq v$ follows from $\tilde{u} \leq v$ in the limit $\varepsilon \downarrow 0$, it will simply suffice to prove that

$$
\tilde{u}(t, x) \leq v(t, x),(t, x) \in(0, T) \times \Omega .
$$

We will assume

$$
\exists(s, z) \in(0, T) \times \Omega \text { and } \tilde{u}(s, z)-v(s, z)=\delta>0
$$

and then contradict this assumption.

By compactness of $[0, T] \times \bar{\Omega} \times \bar{\Omega}$ and upper semicontinuity of $u-v$, and considering $-\frac{\varepsilon}{T-t} \downarrow-\infty$ when $t \uparrow T$, we have $\tilde{u}-v$ is bounded above on $[0, T) \times$ $\bar{\Omega} \times \bar{\Omega}$. Thus there exists $\left(t_{\alpha}, x_{\alpha}, y_{\alpha}\right) \in[0, T) \times \bar{\Omega} \times \bar{\Omega}$ such that it is a maximum point of $\tilde{u}(t, x)-v(t, y)-\frac{\alpha}{2} d^{2}(x, y)$ on $[0, T) \times \bar{\Omega} \times \bar{\Omega}$. We set

$$
m_{\alpha}=\tilde{u}\left(t_{\alpha}, x_{\alpha}\right)-v\left(t_{\alpha}, y_{\alpha}\right)-\frac{\alpha}{2} d^{2}\left(x_{\alpha}, y_{\alpha}\right)
$$

and obviously

$$
m_{\alpha} \geq \tilde{u}(s, z)-v(s, z)-\frac{\alpha}{2} d^{2}(z, z)=\delta>0 .
$$


Let us admit for a moment the following lemma:

\section{Lemma 3.2.}

(i) $t_{\alpha} \neq 0$,

(ii) There exists $\left(t_{0}, x_{0}\right) \in[0, T] \times \Omega$ s.t. when $\alpha \rightarrow+\infty$, along some subsequence which we will still denote by $\left(t_{\alpha}, x_{\alpha}, y_{\alpha}\right)$ s.t. $\left(t_{\alpha}, x_{\alpha}, y_{\alpha}\right) \rightarrow\left(t_{0}, x_{0}, x_{0}\right)$ and $\alpha d^{2}\left(x_{\alpha}, y_{\alpha}\right) \rightarrow 0$.

By Lemma 3.2, we have $t_{\alpha} \in(0, T), x_{\alpha}, y_{\alpha} \in \Omega$ for large $\alpha$.

According to [2], there is $r_{0}>0$ such that for every $x, y \in B\left(x_{0}, r_{0}\right)$, we have that $d(x, y)<\min \left\{i_{M}(x), i_{M}(y)\right\}$, the vectors $\exp _{x}^{-1}(y) \in T M_{x} \equiv T M_{x}^{*}$ and $\exp _{y}^{-1}(x) \in T M_{y} \equiv T M_{y}^{*}$ are well defined, and the function $d^{2}(x, y)$ is $C^{2}$ smooth on $B\left(x_{0}, r_{0}\right) \times B\left(x_{0}, r_{0}\right) \in M \times M$. And we can assume that $x_{\alpha}, y_{\alpha} \in B\left(x_{0}, r_{0}\right)$ for all $\alpha$.

Now, for each $\alpha$, we can apply Theorem 2.7 with $\Omega_{1}=\Omega_{2}=B\left(x_{0}, r_{0}\right), u_{1}=$ $\tilde{u}, u_{2}=-v, \varphi(t, x, y)=\varphi_{\alpha}(x, y)=\frac{\alpha}{2} d^{2}(x, y)$, and for

$$
\varepsilon=\varepsilon_{\alpha}:=\frac{1}{2\left(1+\left\|d^{2} \varphi_{\alpha}\left(x_{\alpha}, y_{\alpha}\right)\right\|\right)} .
$$

Since $\left(t_{\alpha}, x_{\alpha}, y_{\alpha}\right)$ is a global maximum of the function $\tilde{u}(t, x)-v(t, y)-\frac{\alpha}{2} d^{2}(x, y)$ on $(0, T) \times \Omega_{1} \times \Omega_{2}$, the condition (2.1) in Theorem 2.7 is guaranteed by having $\tilde{u}$ (and $v$ ) be a subsolution (resp. supersolution) of a parabolic equation, thus for $\varepsilon=\varepsilon_{\alpha}$ there are numbers $b_{1}, b_{2}$ and bilinear forms $P \in \mathcal{L}_{s}^{2}\left(T M_{x_{\alpha}}\right), Q \in \mathcal{L}_{s}^{2}\left(T M_{y_{\alpha}}\right)$ s.t.

$\left(b_{1}, \frac{\partial}{\partial x} \varphi\left(t_{\alpha}, x_{\alpha}, y_{\alpha}\right), P\right) \in \overline{\mathcal{P}}^{2,+} \tilde{u}\left(t_{\alpha}, x_{\alpha}\right),\left(-b_{2},-\frac{\partial}{\partial y} \varphi\left(t_{\alpha}, x_{\alpha}, y_{\alpha}\right), Q\right) \in \overline{\mathcal{P}}^{2,-} v\left(t_{\alpha}, y_{\alpha}\right)$,

and

$b_{1}+b_{2}=\varphi_{t}\left(t_{\alpha}, x_{\alpha}, y_{\alpha}\right)=0,-\left(\frac{1}{\varepsilon_{\alpha}}+\left\|A_{\alpha}\right\|\right)\left(\begin{array}{cc}I & 0 \\ 0 & I\end{array}\right) \leq\left(\begin{array}{cc}P & 0 \\ 0 & -Q\end{array}\right) \leq A_{\alpha}+\varepsilon_{\alpha} A_{\alpha}^{2}$,

where $A_{\alpha}=d^{2} \varphi\left(t_{\alpha}, x_{\alpha}, y_{\alpha}\right) \in \mathcal{L}_{s}^{2}\left(T(M \times M)_{\left(x_{\alpha}, y_{\alpha}\right)}\right)$. Therefore, according to the assumption, we have that

$F\left(t_{\alpha}, y_{\alpha}, r, \alpha \exp _{y_{\alpha}}^{-1}\left(x_{\alpha}\right), Q\right)-F\left(t_{\alpha}, x_{\alpha}, r,-\alpha \exp _{x_{\alpha}}^{-1}\left(y_{\alpha}\right), P\right) \leq \omega\left(\alpha d^{2}\left(x_{\alpha}, y_{\alpha}\right)+d\left(x_{\alpha}, y_{\alpha}\right)\right)$.

On the other hand, by the conclusion in Section 3 of [2], we have that

$$
\frac{\partial}{\partial x} \varphi\left(t_{\alpha}, x_{\alpha}, y_{\alpha}\right)=-\alpha \exp _{x_{\alpha}}^{-1}\left(y_{\alpha}\right),-\frac{\partial}{\partial y} \varphi\left(t_{\alpha}, x_{\alpha}, y_{\alpha}\right)=\alpha \exp _{y_{\alpha}}^{-1}\left(x_{\alpha}\right)
$$


Combining the definition of viscosity supersolution and subsolution, we have

$$
\begin{aligned}
& b_{1}+F\left(t_{\alpha}, x_{\alpha}, \tilde{u}\left(t_{\alpha}, x_{\alpha}\right)+\frac{\varepsilon}{T-t},-\alpha \exp _{x_{\alpha}}^{-1}\left(y_{\alpha}\right), P\right) \leq-\frac{\varepsilon}{\left(T-t_{\alpha}\right)^{2}} \leq-\frac{\varepsilon}{T^{2}}<0 \\
& -b_{2}+F\left(t_{\alpha}, y_{\alpha}, v\left(t_{\alpha}, y_{\alpha}\right), \alpha \exp _{y_{\alpha}}^{-1}\left(x_{\alpha}\right), Q\right) \geq 0
\end{aligned}
$$

this with properness of $F$ and (3.3), we have

$$
\begin{aligned}
\frac{\varepsilon}{T^{2}} & \leq F\left(t_{\alpha}, y_{\alpha}, v\left(t_{\alpha}, y_{\alpha}\right), \alpha \exp _{y_{\alpha}}^{-1}\left(x_{\alpha}\right), Q\right)-F\left(t_{\alpha}, x_{\alpha}, \tilde{u}\left(t_{\alpha}, x_{\alpha}\right)+\frac{\varepsilon}{T-t},-\alpha \exp _{x_{\alpha}}^{-1}\left(y_{\alpha}\right), P\right) \\
& \leq F\left(t_{\alpha}, y_{\alpha}, \tilde{u}\left(t_{\alpha}, x_{\alpha}\right), \alpha \exp _{y_{\alpha}}^{-1}\left(x_{\alpha}\right), Q\right)-F\left(t_{\alpha}, x_{\alpha}, \tilde{u}\left(t_{\alpha}, x_{\alpha}\right),-\alpha \exp _{x_{\alpha}}^{-1}\left(y_{\alpha}\right), P\right) \\
& \leq \omega\left(\alpha d^{2}\left(x_{\alpha}, y_{\alpha}\right)+d\left(x_{\alpha}, y_{\alpha}\right)\right) .
\end{aligned}
$$

This is a contradiction by Lemma 2.2 if we let $\alpha$ tend to $\infty$.

The proof of Lemma 3.2:

(i)If $t_{\alpha}=0$, we have

$0<\delta \leq m_{\alpha}=\psi\left(x_{\alpha}\right)-\psi\left(y_{\alpha}\right)-\frac{\alpha}{2} d^{2}\left(x_{\alpha}, y_{\alpha}\right)-\frac{\varepsilon}{T} \leq \sup _{\bar{\Omega} \times \bar{\Omega}}\left(\psi(x)-\psi(y)-\frac{\alpha}{2} d^{2}(x, y)\right)-\frac{\varepsilon}{T}$.

However, since $\psi \in C(\bar{\Omega})$, the right-hand side above tends to $-\frac{\varepsilon}{T}$ according to Lemma 4.1 in [1], so $t_{\alpha} \neq 0$ if $\alpha$ is large.

(ii) Since $\left(t_{\alpha}, x_{\alpha}, y_{\alpha}\right)$ is a sequence in a compact set $[0, T] \times \bar{\Omega} \times \bar{\Omega}$, there must be some subsequence which we still denote $\left(t_{\alpha}, x_{\alpha}, y_{\alpha}\right)$ that is convergent to a limit $\left(t_{0}, x_{0}, y_{0}\right) \in[0, T] \times \bar{\Omega} \times \bar{\Omega}$ when $\alpha \rightarrow+\infty$. If $x_{0} \neq y_{0}, \alpha d^{2}\left(x_{\alpha}, y_{\alpha}\right)$ will tends to $+\infty$. Combining that $\tilde{u}-v$ is upper bounded, we get $m_{\alpha} \rightarrow-\infty$, which is also a contradiction to $m_{\alpha} \geq \delta>0$.

When $t_{\alpha} \uparrow T, m_{\alpha} \rightarrow-\infty$, so $t_{0} \neq T$. If $x_{0}=y_{0} \in \partial \Omega$, by the upper semicontinuity of $\tilde{u}-v$, we have

$$
\begin{aligned}
0<\delta \leq \varlimsup_{\alpha \rightarrow \infty} m_{\alpha} & =\varlimsup_{\alpha \rightarrow \infty}\left(\tilde{u}\left(t_{\alpha}, x_{\alpha}\right)-v\left(t_{\alpha}, y_{\alpha}\right)-\frac{\alpha}{2} d^{2}\left(x_{\alpha}, y_{\alpha}\right)\right) \\
& \leq \lim _{\alpha \rightarrow \infty}\left(\tilde{u}\left(t_{\alpha}, x_{\alpha}\right)-v\left(t_{\alpha}, y_{\alpha}\right)\right) \\
& \leq \tilde{u}\left(t_{0}, x_{0}\right)-v\left(t_{0}, x_{0}\right) \leq \frac{\varepsilon}{t-T}-0<0
\end{aligned}
$$

which is obviously a contradiction, so $x_{0} \in \Omega$.

Since $\tilde{u}-v$ is bounded above, there exists a constant $C$ s.t.

$$
0<\delta \leq m_{\alpha}=\tilde{u}\left(t_{\alpha}, x_{\alpha}\right)-v\left(t_{\alpha}, y_{\alpha}\right)-\frac{\alpha}{2} d^{2}\left(x_{\alpha}, y_{\alpha}\right) \leq \tilde{u}\left(t_{\alpha}, x_{\alpha}\right)-v\left(t_{\alpha}, y_{\alpha}\right) \leq C .
$$

So there exists a subsequence such that $m_{\alpha}$ converges. According to Proposition 3.7 in [1], we have $\alpha d^{2}\left(x_{\alpha}, y_{\alpha}\right) \rightarrow 0$.

Remark 3.3. (see [2])If $M$ has nonnegative sectional curvature, then condition (3.2) implies that $P \leq L_{y x}(Q)$. 
Therefore, if $M$ has nonnegative curvature and $F$ is degenerate elliptic for each fixed $t$, then (3.2) automatically implies that

$$
F\left(t, x, r,-\alpha \exp _{x}^{-1}(y), L_{y x} Q\right)-F\left(t, x, r,-\alpha \exp _{x}^{-1}(y), P\right) \leq 0 .
$$

hence

$$
\begin{aligned}
& F\left(t, y, r, \alpha \exp _{y}^{-1}(x), Q\right)-F\left(t, x, r,-\alpha \exp _{x}^{-1}(y), P\right) \\
= & F\left(t, y, r, \alpha \exp _{y}^{-1}(x), Q\right)-F\left(t, x, r,-\alpha \exp _{x}^{-1}(y), L_{y x} Q\right) \\
& +F\left(t, x, r,-\alpha \exp _{x}^{-1}(y), L_{y x} Q\right)-F\left(t, x, r,-\alpha \exp _{x}^{-1}(y), P\right) \\
\leq & F\left(t, y, r, \alpha \exp _{y}^{-1}(x), Q\right)-F\left(t, x, r,-\alpha \exp _{x}^{-1}(y), L_{y x} Q\right),
\end{aligned}
$$

and we see that the main condition (3.1) in Theorem 3.1 is satisfied if we automatically require, for instance, that

$$
F\left(t, y, r, \alpha \exp _{y}^{-1}(x), Q\right)-F\left(t, x, r,-\alpha \exp _{x}^{-1}(y), L_{y x} Q\right) \leq \omega\left(\alpha d^{2}(x, y)+d(x, y)\right) .
$$

So we only need that $F$ is intrinsically uniformly continuous w.r.t. $x$ uniformly in $t$ (see [2]).

Definition 3.4. We will say that $F \in C(\chi, R)$ is intrinsically uniformly continuous w.r.t. $x$ uniformly in $t$, if

$$
\sup _{t \in(0, T)}\left|F\left(t, y, r, L_{x y} \zeta, L_{x y} P\right)-F(t, x, r, \zeta, P)\right| \rightarrow 0 \text { uniformly as } y \rightarrow x .
$$

Let us sum up what we have just shown:

Corollary 3.5. Let $\Omega$ be a bounded open subset of a complete finitedimensional Riemannian manifold $M$ with nonnegative sectional curvature, and $F \in C(\chi, R)$ be continuous, proper for each fixed $t \in(0, T)$ and intrinsically uniformly continuous w.r.t. $x$ uniformly in $t$.

Let $u \in U S C([0, T) \times \bar{\Omega})$ be a subsolution and $v \in L S C([0, T) \times \bar{\Omega})$ a supersolution of (1.1). Then $u \leq v$ on $[0, T) \times \Omega$.

In particular PDEs (1.1) has at most one viscosity solution.

When $M$ has negative curvature, the main condition (3.1) in Theorem 3.1 involves kind of a uniform continuity assumption on the dependence of $F$ with respect to $d^{2} u(t, x)$ by virtue of the following remark(see [2]).

Remark 3.6. Assume that $M$ has sectional curvature bounded below by some constant $-K_{0} \leq 0$. Then condition (3.2) in Theorem 3.1 implies that

$$
P-L_{y x}(Q) \leq \frac{3}{2} K_{0} \alpha d^{2}(x, y) I,
$$


where $I(v, v)=\|v\|^{2}$.

Corollary 3.7. Let $M$ be a compact Riemannian manifold (no assumption on curvature) and $\Omega$ is a bounded open subset of $M$. Suppose that $F \in C(\chi, R)$ is proper, continuous, and satisfies the following uniform continuity assumption: for every $\varepsilon>0$, there exists $\delta>0$ such that

$d(x, y) \leq \delta, P-L_{y x}(Q) \leq \delta I \Rightarrow \sup _{t \in(0, T)}\left\{F\left(t, y, r, \alpha \exp _{y}^{-1}(x), Q\right)-F\left(t, x, r,-\alpha \exp _{x}^{-1}(y), P\right)\right\} \leq \varepsilon$

for each fixed $t$ and for all $x, y \in \Omega$ with $d(x, y)<i(M), r \in R, \alpha>0, P \in$ $\mathcal{L}_{s}^{2}\left(T M_{x}\right)$ and $Q \in \mathcal{L}_{s}^{2}\left(T M_{y}\right)$. Then there is at most one viscosity solution to PDEs (1.1).

Remind that $M$ has sectional curvature bounded below since it is compact, so the conclusion is an analogue of Corollary 4.10 in [2]. We omit the proof.

\section{Existence result}

In [1], detailed research called Perron's method has been adapted to establish existence of viscosity solution to the Dirichlet problem in Euclidean space. The same method has been used to get the existence result for Dirichlet problem on Riemannian manifolds. For our PDEs (1.1), we can go exactly as in [1] with appropriate changes to get our result as follows:

Theorem 4.1. Let comparison hold for (1.1), i.e., if $\omega$ is a subsolution of (1.1) and $v$ is a supersolution of (1.1), then $\omega \leq v$. Suppose also that there exists a subsolution $\underline{\underline{u}}$ and a supersolution $\bar{u}$ of (1.1) that satisfy the initial condition $\underline{\mathrm{u}}_{*}(0, x)=\bar{u}^{*}(0, x)=\psi(x)$ for $x \in \bar{\Omega}$ and $\underline{\mathrm{u}}_{*}(t, x)=\bar{u}^{*}(t, x)=h(t, x)$ for $(t, x) \in$ $[0, T) \times \partial \Omega$. Then

$$
W(t, x)=\sup \{\omega(t, x): \underline{u} \leq \omega \leq \bar{u} \text { and } \omega \text { is a subsolution of }(1.1)\}
$$

is a solution of (1.1).

Here we used the following notation:

$$
\begin{aligned}
& u^{*}(t, x)=\lim _{r \downarrow 0} \sup \{u(s, y):(s, y) \in(0, T) \times M \text { and }|s-t| \leq r, d(y, x) \leq r\} ; \\
& u_{*}(t, x)=\lim _{r \downarrow 0} \inf \{u(s, y):(s, y) \in(0, T) \times M \text { and }|s-t| \leq r, d(y, x) \leq r\},
\end{aligned}
$$

that is $u^{*}$ denotes the upper semicontinuous envelope of $u$ (the smallest upper semicontinuous function, with values in $[-\infty,+\infty]$, satisfying $\left.u \leq u^{*}\right)$, and similarly $u_{*}$ stands for the lower semicontinuous envelope of $u$.

As in [1], we need the following several steps which are Riemannian versions for parabolic PDEs. 
Proposition 4.2. Let $\Omega \subset M$ be locally compact, $u \in U S C([0, T) \times \bar{\Omega})$, $(t, z) \in(0, T) \times \Omega$, and $(p, \zeta, A) \in \mathcal{P}^{2,+} u(t, z)$. Suppose that $u_{n}$ is a sequence of upper semicontinuous functions defined on $[0, T) \times \bar{\Omega}$ s.t.

(i)there exists $\left(t_{n}, x_{n}\right) \in(0, T) \times \Omega$ such that $\left(t_{n}, x_{n}, u_{n}\left(t_{n}, x_{n}\right)\right) \rightarrow(t, z, u(t, z))$, (ii)if $\left(s_{n}, y_{n}\right) \in(0, T) \times \Omega$ and $\left(s_{n}, y_{n}\right) \rightarrow(s, y) \in(0, T) \times \Omega$,

then $\lim \sup _{n \rightarrow \infty} u_{n}\left(s_{n}, y_{n}\right) \leq u(s, y)$.

Then there exist $\left(\hat{t}_{n}, \hat{x}_{n}\right) \in(0, T) \times \Omega$ and $\left(p_{n}, \zeta_{n}, A_{n}\right) \in \mathcal{P}^{2,+} u_{n}\left(t_{n}, z_{n}\right)$ such that

$$
\left(\hat{t}_{n}, \hat{x}_{n}, u_{n}\left(\hat{t}_{n}, \hat{x}_{n}\right), p_{n}, \zeta_{n}, A_{n}\right) \rightarrow(t, z, u(t, z), p, \zeta, A) .
$$

Proof. The Euclidean version of the above proposition for elliptic functions was proved in [1]. Azagra et al. generalized it to the setting of Riemannian manifolds. For Euclidean version of parabolic functions, we can refer you to [4]. If we note Remark 2.3, we can also generalized the result in [4] to get our proof for the above Proposition with the same method as in [2]. So we omit the proof.

Use the above proposition, we can soon get the following lemma just like in [1]:

Lemma 4.3. Let $\Omega \in M$ be locally compact and $F \in C(\chi, R)$ be continuous for each fixed $t \in(0, T)$. $\mathcal{F}$ is a family of solution of $u_{t}+F \leq 0$ in $(0, T) \times \Omega$. Let $\omega(t, x)=\sup \{u(t, x): u \in \mathcal{F}\}$ and assume that $\omega^{*}(t, x)<\infty$ for $(t, x) \in$ $(0, T) \times \Omega$. Then $\omega^{*}$ is a solution of $u_{t}+F \leq 0$ in $(0, T) \times \Omega$.

The following lemma is also important to the existence result.

Proposition 4.4. Let $\Omega \subset M$ be open and $u$ be solution of $u_{t}+F \leq 0$ in $(0, T) \times \Omega$. If $u_{*}$ fails to be a supersolution at some point $(\hat{t}, \hat{x})$, i.e., there exists $(p, \zeta, A) \in \mathcal{P}^{2,-} u_{*}(\hat{t}, \hat{x})$ for which $p+F\left(\hat{t}, \hat{x}, u_{*}(\hat{t}, \hat{x}), \zeta, A\right)<0$, then for any small $\kappa>0$ there is a subsolution $u_{\kappa}$ of $u_{t}+F \leq 0$ in $(0, T) \times \Omega$ satisfying

$$
\left\{\begin{array}{l}
u_{\kappa}(t, x) \geq u(t, x) \text { and } \sup _{(0, T) \times \Omega}\left(u_{\kappa}-u\right)>0, \\
u_{\kappa}(t, x)=u(t, x) \text { for }(t, x) \in(0, T) \times \Omega,|t-\hat{t}|+d(x, \hat{x}) \geq \kappa
\end{array}\right.
$$

Proof. Set

$$
\varphi_{\delta, \gamma}(t, v)=u_{*}(\hat{t}, \hat{x})+\delta+p(t-\hat{t})+\langle\zeta, v\rangle_{\hat{x}}+\frac{1}{2}\langle A v, v\rangle_{\hat{x}}-\gamma\|v\|^{2}-\gamma(t-\hat{t}) .
$$

Then, by continuity, $\exists r, \delta, \gamma>0$ small enough s.t. in

$$
B_{r}=\{(t, v):|t-\hat{t}|+\|v\|<r\},
$$

we have

$$
p-\gamma+F\left(t, \exp _{\hat{x}}(v), \varphi_{\delta, \gamma}(t, v), \zeta-2 \gamma v, A-2 \gamma I\right)<0 .
$$


Set $x=\exp _{\hat{x}}(v)$. Since $(p, \zeta, A) \in \mathcal{P}^{2,-} u_{*}(\hat{t}, \hat{x})$, by Proposition 2.2 we have $u(t, x) \geq u_{*}(t, x)=u_{*}\left(t, \exp _{\hat{x}}(v)\right) \geq u_{*}(\hat{t}, \hat{x})+p(t-\hat{t})+\langle\zeta, v\rangle_{\hat{x}}+\frac{1}{2}\langle A v, v\rangle_{\hat{x}}-o\left(\|v\|^{2}+|t-\hat{t}|\right)$,

if we choose $\delta=\frac{r^{2}}{8} \gamma$ then $u(t, x)>\varphi_{\delta, \gamma}\left(t, \exp _{\hat{x}}^{-1}(x)\right):=u_{\delta, \gamma}(t, x)$ for $\frac{r}{2} \leq\|v\| \leq r$ if $r$ is sufficiently small.

According to Proposition 2.8 in [2], we have

$$
d \varphi_{\delta, \gamma}\left(t, 0_{\hat{x}}\right)=d u_{\delta, \gamma}(t, \hat{x}), d^{2} \varphi_{\delta, \gamma}\left(t, 0_{\hat{x}}\right)=d^{2} u_{\delta, \gamma}(t, \hat{x}),
$$

and

$$
\frac{\partial \varphi_{\delta, \gamma}}{\partial t}=\frac{\partial u_{\delta, \gamma}}{\partial t}, \lim _{v \rightarrow 0_{\hat{x}}} d \varphi_{\delta, \gamma}(t, v)=\lim _{x \rightarrow \hat{x}} d u_{\delta, \gamma}(t, x), \lim _{v \rightarrow 0_{\hat{x}}} d^{2} \varphi_{\delta, \gamma}(t, v)=\lim _{x \rightarrow \hat{x}} d^{2} u_{\delta, \gamma}(t, x),
$$

in a neighborhood of $(\hat{t}, \hat{x})$.

So there exists $\kappa>0$ small enough s.t. $u_{\delta, \gamma} \in C^{1,2}\left(B_{\kappa}\right)$, where $B_{\kappa}=\{(t, x)$ : $|t-\hat{t}|+d(x, \hat{x})<\kappa\}$. Moreover, $u(t, x)>u_{\delta, \gamma}(t, x)$ and $u_{\delta, \gamma}(t, x)$ is a solution of $u_{t}+F \leq 0$ when $(t, x) \in B_{\kappa}$. Then, by Lemma 4.3, the function

$$
u_{\kappa}(t, x)= \begin{cases}\max \left(u(t, x), u_{\delta, \gamma}(t, x)\right), & (t, x) \in B_{\kappa}, \\ u(t, x) & \text { otherwise, }\end{cases}
$$

is a solution of $u_{t}+F \leq 0$ in $(0, T) \times \Omega$. The last observation is that in every neighborhood of $(\hat{t}, \hat{x})$ there are points such that $u_{\kappa}(t, x)>u(t, x)$; indeed, by definition, there is sequence $\left(t_{n}, x_{n}, u\left(t_{n}, x_{n}\right)\right)$ convergent to $\left(\hat{t}, \hat{x}, u_{*}(\hat{t}, \hat{x})\right)$ and then

$$
\lim _{n \rightarrow \infty}\left(u_{\kappa}\left(t_{n}, x_{n}\right)-u\left(t_{n}, x_{n}\right)\right)=u_{*}(\hat{t}, \hat{x})+\delta-u_{*}(\hat{t}, \hat{x})>0
$$

Having these above preparation, we can easily achieve our proof of Theorem 4.1 as in [1]. We omit it.

\section{References}

[1] M.G. Crandall, H. Ishii, P.-L. Lions, User's guide to viscosity solutions of second order partial differential equations, Bull, Amer. Math. Soc. 27 (1992) no. 1, 1-67.

[2] D. Azagra, J. Ferrera, B. Sanz, Viscosity solutions to second order partial differential equations on Riemannian manifolds, Journal of Differential Equations, Vol. 245(2008), 307-336. 
[3] D. Azagra, M. Jimenez-Sevilla, F. Macia, Generalized motion of level sets by functions of their curvatures on Riemannian manifolds, Calculus of Variations and PDE 33 (2008), 133-167.

[4] M.P. do Carmo, Riemannian Geometry, Mathematics: Theory and Applications, Birkhäuser Boston, 1992.

[5] W. Liu, Y. Yang, G. Lu, Viscosity solutions of fully nonlinear parabolic systems, Journal of Mathematical Analysis and Applications, Vol. 281(2003) no.1, 362-381. 